\title{
Recovery of an Initial Temperature from Discrete Sampling
}

\author{
Ronald DeVore and Enrique Zuazua*
}

January 20, 2014

\begin{abstract}
The problem of recovering the initial temperature of a body from discrete temperature measurements made at later times is studied. While this problem has a general formulation, the results of this paper are only given in the simplest setting of a finite (one dimensional), constant coefficient, linear rod. It is shown that with a judicious placement of a thermometer on this rod, the initial temperature profile of the rod can be completely determined by later time measurements. The paper then studies the number of measurements that are needed to recover the initial profile to a prescribed accuracy and provides an optimal reconstruction algorithm under the assumption that the initial profile is in a Sobolev class.
\end{abstract}

\section{Introduction}

This paper addresses the classical problem of Inverse Heat Conduction (IHC), see [1]. More precisely, we are interested in how accurately we can recover the initial temperature distribution of a finite body from temperature measurements made at a fixed number $n$ of later times. Such measurements correspond to placing one or more thermometers at specified physical locations and then recording the temperatures at a sequence of times $t_{1}, \ldots, t_{n}$ with $t_{1}<t_{2}<\cdots<t_{n}$.

We address this problem in the context of the heat equation as a prototype of parabolic highly time-irreversible PDEs. In this context, the problem of recovering the initial datum leading to the evolution, out of future measurements, is well-known to be highly ill-posed.

This problem has been intensively investigated. We refer to the book [3] for a rather complete and updated presentation of the state of the art in parabolic inverse problems theory. But, most often, the reconstruction of the initial datum is addressed by means of partial measurements on the solutions in continuous time. We refer for instance to [14] where the initial datum is recovered in $L_{2}$ from measurements of the normal derivative of the solutions in continuous time.

The inverse problem under consideration is not only relevant in the context of heat propagation ([1]), but in many other fields where the heat equation plays a central role. This is particularly the

${ }^{*}$ This research was initiated when the first author was a visiting scholar at the Basque Center for Applied Mathematics, in the frame of the NUMERIWAVES AdvG of ERC. This research was supported by the Office of Naval Research Contracts ONR N00014-09-1-0107, ONR N00014-11-1-0712, ONR N00014-12-1-0561; and the National Science Foundation Grant DMS 12 22390, This publication is based on work supported by Award No. KUS-C1-016-04, made by King Abdullah University of Science and Technology (KAUST). The work of the second author was supported by Grant MTM2011-29306-C02-00 of the MICINN (Spain), the Advanced Grant FP7-246775 of the European Research Council Executive Agency and the Grant PI2010-04 of the Basque Government. This work was finished while the second author was visiting the Laboratoire Jacques Louis Lions with the support of the Paris City Hall "Research in Paris" program. 
case in many contexts of population dynamics and Mathematical Biology, see [13]. The techniques we shall develop can also be employed for other relevant time-irreversible processes such as the system of viscoelasticity $([6])$ or parabolic equations with memory effects $([11])$.

In this paper we address the problem of the one dimensional linear heat equation in a bounded interval with Dirichlet boundary conditions

$$
u_{t}=u_{x x}, \quad u(0, t)=u(\pi, t)=0, \quad u(x, 0)=f(x)
$$

whose solution can be represented in Fourier series,

$$
u(x, t)=\sum_{k=1}^{\infty} \hat{f}_{k} e^{-k^{2} t} \sin k x
$$

where $\hat{f}_{k}$ are the Fourier sine coefficients of $f$. We consider the case of a single thermometer placed at a fixed position $x_{0}$. In other words, we analyze the problem of reconstructing the initial datum $f=f(x)$ out of the values of the solution at some given $x=x_{0}$ in the time-instances $t_{1}, \ldots, t_{n}$ with $t_{1}<t_{2}<\cdots<t_{n}$.

It is important to underline that the inverse problem we consider is not overdetermined since we are trying to recover a 1-d space dependent function (the initial datum) out of the time-evolution of the solution at $x_{0}$, which is a $1-d$ function as well. Note also that, for the same reasons, the corresponding multi-dimensional version of the problem would be underdetermined.

Employing the Fourier series representation above, we present an algorithm for reconstructing $f$ from measurements taken at different time instances. Under the assumption that the initial profile is in a Sobolev class, we determine the number of measurements and the precise timeinstances that are needed to recover the initial profile to a prescribed accuracy. Of course, once the initial temperature is properly approximated, the complete dynamics can be recovered by classical numerical approximation procedures.

The first question to be addressed is where to place the thermometer, i.e. its position $x_{0}$ on the rod, and at which times to make the measurements. Once the measurement point $x_{0}$ is chosen we can write

$$
u\left(x_{0}, t\right)=\sum_{k=1}^{\infty} \hat{f}_{k} e^{-k^{2} t} \sin k x_{0},
$$

in the spirit of Dirichlet series ([15]). Accordingly, the position $x_{0}$, of course, has to be chosen in a strategic manner, to ensure that $\sin k x_{0} \neq 0$ for all $k$, and thereby avoid the nodal sets of the eigenfunctions. Indeed, in case the thermometer were placed within the nodal set of some eigenfunction, this eigen-component would be invisible in the expansion (1.3) of $u\left(x_{0}, t\right)$ and therefore the energy of the initial temperature on that mode would not be recoverable, regardless of the number of choice of the sampling time-instances.

The need of employing strategic points to observe or control heat processes is well-known in the context of point-wise control of parabolic equations, see [8]. In the one dimensional case under consideration, this amounts to choosing $x_{0}$ satisfying a suitable irrationality condition. But, of course, in the multi-dimensional case it is a generic property that is harder to check and depends in particular on the geometry of the domain under consideration.

Our methods, being based on Fourier series expansions, can be extended to many other situations, including variable coefficients and multi-dimensional problems. But, as mentioned above, the choice of the placement of the thermometer depends on the nodal sets of the eigenfunctions. 
On the other hand, the selection of the time instances in which the observation will be made depends critically on the distribution of eigenvalues. As we shall see, our analysis is based on the fact that the eigenvalues of the one dimensional problem are distributed along a parabola and this determines the optimal choice of the sampling instances. Since this issue is extremely sensitive, it would be interesting to see what happens for other spectra, for instance for the heat equation on the whole line where the use of similarity variables reduces the problem to a parabolic equation with an explicit spectrum growing linearly (see [10] for an application of this idea in the context of the null control of the heat equation).

Our method could also be applied to the case where the sensor would be placed in the boundary of the rod. In that case, of course, the quantity to be measured would be the normal derivative of the solution, i. e. the heat flux through the boundary. For instance when placing the sensor at the boundary point $x=0$ the corresponding normal derivative takes the form

$$
u_{x}(0, t)=\sum_{k=1}^{\infty} k \hat{f}_{k} e^{-k^{2} t},
$$

comparable to (1.3). Of course, in this case, the strategic character of the placement of the sensor is guaranteed since normal derivatives of the eigenfunctions do not degenerate, leading to the weights $k$ in (1.4), instead of $\sin k x_{0}$.

Our techniques cannot be applied for nonlinear problems, or even for linear non-autonomous ones. Obtaining sharp reconstruction algorithms in this setting would require completely different ideas and this constitutes a challenging problem.

Let us now explain in more detail the main results and methods developed in the paper. We first address the problem of consistency (see Section §2). It is shown that if the thermometer is placed at a suitable (strategic) location $x_{0}$, then it is indeed possible to recover $f$ from an infinite number of discrete measurements made at times $t_{1}<t_{2}<\ldots$ This is in agreement with the fact that, as mentioned above, the problem under consideration is properly determined, without being either under or over-determined.

The following sections of the paper consider the problem of how accurately we can approximate $f$ from a fixed budget $n$ of temperature measurements made at times $t_{1}<t_{2}<\cdots<t_{n}$. Note that if we have no a priori information about $f$, other than it being a function in $L_{2}:=L_{2}[0, \pi]$, then we cannot guarantee any specific rate of accuracy in the performance of any measurement scheme, regardless what the choice of the time-instances $t_{j}$ is. This leads us to stipulate that $f$ comes from a compact class $\mathcal{F}$ of $L_{2}$.

Various compact classes are possible but the most natural are to assume some smoothness about $f$ that corresponds to membership in a Sobolev or Besov class. We shall only consider the case where $\mathcal{F}=\mathcal{F}_{r}$ is a fixed ball of the Sobolev space $W^{r}\left(L_{2}\right)$. We define this ball by the restriction

$$
\sum_{k=1}^{\infty} k^{2 r}\left|\hat{f}_{k}\right|^{2} \leq 1 .
$$

Under this constraint for some $r>0$, we will consider the recovery of $f$ in the $L_{2}$ norm and we shall actually build an algorithm and prove it is optimal in terms of rate distortion (error versus the number of measurements).

So our problem is that, $f$ being as in (1.5), we are given a budget $n$ and are allowed to ask for $n$ later temperatures of $u$ all taken at a fixed point $x_{0} \in(0, \pi)$ and at different times $t_{1}, \ldots, t_{n}$. That 
is, we ask for the values $u_{j}: u\left(x_{0}, t_{j}\right), j=1, \ldots, n$ each of them leading to the following infinite combination of all the Fourier coefficients of $f$ :

$$
u\left(x_{0}, t_{j}\right)=\sum_{k=1}^{\infty} \hat{f}_{k} e^{-k^{2} t_{j}} \sin k x_{0} .
$$

Once the selection of the point $x_{0}$ and the times $t_{1}, \ldots, t_{n}$ is specified, then the taking of the measurements $u_{j}:=u\left(x_{0}, t_{j}\right), j=1, \ldots, n$ (by an encoder or sensor), extracts information about $f$. To construct an approximation to $f$, one employs a decoder which uses the values $u_{j}, j=1, \ldots, n$, and the knowledge of what $x_{0}$ and the $t_{j}$ 's are, to create an approximation to $f$. We view the decoder as a mapping

$$
A_{n}:\left(x_{0} ; t_{1}, \ldots, t_{n} ; u_{1}, \ldots, u_{n}\right) \longrightarrow L_{2},
$$

which takes the given data and creates the approximation $\bar{f}_{n}:=A_{n}\left(x_{0} ; t_{1}, \ldots, t_{n} ; u_{1}, \ldots, u_{n}\right)$ to $f$ from these values. In order to impose numerical stability of the scheme, we require that $A_{n}$ is continuous with respect to the data, i.e. as a mapping from $\mathbb{R}^{n}$ into $L_{2}$.

A measurement algorithm is an encoder coupled with a decoder, i.e. a continuous mapping $A_{n}$ as described above. We are interested in the performance of such a measurement algorithm in terms of the budget $n$ of measurements. For such a measurement scheme, we define its performance by

$$
\hat{\delta}_{n}\left(\mathcal{F}_{r}, L_{2}\right):=\sup _{f \in \mathcal{F}_{r}}\left\|f-\bar{f}_{n}\right\|_{L_{2}}
$$

That is, we consider its performance on the worst of the $f \in \mathcal{F}_{r}$.

There are two settings that one can consider for the times at which to make measurements. The first is to choose once and for all the times $t_{1}, \ldots, t_{n}$ depending only on the class $\mathcal{F}=\mathcal{F}_{r}$. We call this the fixed times problem. The second possibility is to allow the times to be chosen depending on the particular $f \in \mathcal{F}$. Since $f$ is unknown, one would use the previous temperature measurements to decide on when to make the next measurement. We call this type of scheme adaptive.

Notice that in a practical setting, one would not be able to make time measurements too close to one another since the thermometer would have to recalibrate. Also note that even in an adaptive scheme, the first time $t_{1}$ for measurement is fixed independent of $f$.

In $\S 3$, we prove there is a fixed constant $c_{r}$ such that

$$
\hat{\delta}_{n}\left(\mathcal{F}_{r}\right) \geq c_{r} n^{-r}, \quad n=1,2, \ldots,
$$

where the constant $c_{r}$ depends only on $r$. This result applies equally well to the adaptive and fixed time algorithms. This means that, regardless of the choice of the time-instances in which the measurements are done, we can not guarantee that $f$, under the constraint (1.5), is approximated with $L_{2}$ error better than the order $n^{-r}$.

Having this bound on best possible performance, we turn to the study of whether the rate $n^{-r}$ can be achieved and if so where to place the thermometer and when to make the time measurements in order to achieve rate optimal performance. In $\S 4$, choosing $x_{0}$ to be an algebraic number of second order, we give a sequence $t_{1}<t_{2}<\ldots$, such that each $t_{1}, \ldots, t_{n}$ gives a selection of times which achieve the rate $O\left(n^{-r}\right)$ on $\mathcal{F}_{r}$, for each $n$. An important point to stress is that the choice of times at which to make the measurements does not depend on the value of $r$ and therefore do not need knowledge of this value. The value of $r$ only enters into the picture when we analyze the 
performance of the algorithm. In the final section of the paper, we mention some open questions related to this recovery problem.

The literature on the ill-posedness of the backward heat equation and its numerical resolution is huge and goes back, at least, to the work by F. John [7]. The classical literature of inverse problems for heat equations fully describes the ill-posedness of the problem to later build numerical algorithms by means of regularization procedures (see for instance [7]). The approach we undertake is of a different nature, and closer to [9]. We do not intend to fully describe the ill-posedness of the problem but, rather, assuming it is unavoidable, we directly build an algorithm that provides the best possible approximation rates. The extension of this approach to a more general class of parabolic equations is a challenging topic with important potential applications that we discuss in the final section.

\section{Placement of the thermometer and consistency}

We begin by proving that it is indeed possible to select a position $x_{0} \in[0, \pi]$, for the thermometer, and a sequence of times $t_{1}<t_{2}<\ldots$ such that the values $u_{i}:=u\left(x_{0}, t_{i}\right), i=1,2, \ldots$, uniquely determine $f$ whenever $f \in L_{2}$.

Given the representation (1.2), we see that the position $x_{0}$ where we place the thermometer must have the property $\sin k x_{0} \neq 0$ for all $k \geq 1$. In later considerations, it will be important to keep the values $\sin k x_{0}, k \geq 1$, as far from zero as possible. To accomplish this, we let $\alpha \in(0,1)$ be an algebraic number of second order so that

$$
\operatorname{dist}\left(\alpha,\left\{0, \frac{1}{m}, \ldots, \frac{m-1}{m}, 1\right\}\right) \geq \frac{c_{0}}{m^{2}}, \quad m=1,2, \ldots,
$$

with $c_{0}$ an absolute constant. We then take $x_{0}:=\alpha \pi$ and fix $x_{0}$ for the remainder of this paper. It follows that

$$
\operatorname{dist}\left(k x_{0},\{0, \pi, \ldots, k \pi\}\right) \mid \geq c_{0} \pi k^{-1}, \quad k \geq 1 .
$$

and therefore,

$$
\left|\sin k x_{0}\right| \geq d_{0} k^{-1}, \quad k \geq 1
$$

with $d_{0}$ an absolute constant. ${ }^{1} 2$

We will next show that for any choice of the sequence $t_{j}$, such that $t_{j}$ tends to $\infty$, the values of $u_{j}, j=1, \ldots$ uniquely determine the initial datum $f$. For any given $f$, it is convenient to introduce the function

$$
F_{0}(z):=\sum_{k=1}^{\infty} c_{k} z^{k^{2}}
$$

with

$$
c_{k}:=\hat{f}_{k} \sin k x_{0}, \quad k=1,2, \ldots
$$

Since the sequence $\left(c_{k}\right)$ is in $\ell_{2}$, the function $F_{0}$ is analytic in the unit complex disc $D:=\{z:|z|<$ $1\}$.

\footnotetext{
${ }^{1}$ The analysis of this paper could be also extended to other classes of strategic points $x_{0}$ where $\sin k x_{0} \neq 0$ for all $k \geq 1$ but so that $(2.2)$ is not satisfied. It would be interesting to systematically analyze the optimal choice of the measurement time-instances $t_{j}$ as a function of the strategic placement $x_{0}$.

${ }^{2}$ Note that the case where the measurement is done through the boundary, as mentioned in the introduction, is covered fully in this setting since, in that case, the corresponding weight is $k$, which obviously fulfills (2.2).
} 
The importance of $F_{0}$ to us is that $F_{0}\left(e^{-t}\right)=u\left(x_{0}, t\right)$ for any $t>0$. If we ask for the values $u_{j}:=u\left(x_{0}, t_{j}\right), j=1,2, \ldots$, we receive the values $F_{0}\left(z_{j}\right)$ where $z_{j}:=e^{-t_{j}^{2}}$. These values uniquely determine $F_{0}$. Indeed, if $g$ is another function giving rise to $G_{0}$ and $F_{0}=G_{0}$ at the points $z_{j}$, $j=1,2 \ldots$, then $F_{0}-G_{0}$ is analytic and vanishes at a sequence of points which has a limit point in $D$. Hence $F_{0}-G_{0} \equiv 0$ and $F_{0}$ is therefore uniquely determined by these values. This means that the $\left(c_{k}\right)_{k \geq 1}$ are uniquely determined by the values $\left(u_{j}\right)_{j \geq 1}$. Since $\hat{f}_{k}=c_{k} / \sin k x_{0}$ and $\sin k x_{0}$ is never zero, the values $u_{j}$ uniquely determine $f$.

This shows that any choice of the sequence $t_{j}$ so that $t_{j} \rightarrow \infty$ ensures the uniqueness of $f$. But this argument is far from sufficient to provide any quantitative estimate of the degree of approximation of $f$ achieved out of $n$ measurements at $t_{1}, \ldots, t_{n}$.

One could utilize the analyticity of $F$ to give specific formulas on how to recover the values $\hat{f}_{k}$, $k \geq 1$, from the values $\left(u_{j}\right)$ but we shall not derive these since it is not possible to prove any a priori bounds on the performance of such recovery schemes without additional assumptions on $f$. In fact, in [15], Section 2.9, using Dirichlet series, explicit reconstruction formulas are given, but they turn out to be rather unstable. We discuss the recovery for $f \in \mathcal{F}_{r}$ below.

\section{Bounds on optimal performance}

To establish the lower bound (1.9) for optimal performance, we use the theory of nonlinear widths. We recall the notion of manifold width that was introduced in [4]. In this setting, to approximate the elements of the compact set $\mathcal{F}$, one allows any approximation scheme of the following type. There are two continuous mappings $a$ and $M$. The first mapping $a$ takes any $f \in \mathcal{F}$ and maps it into $\mathbb{R}^{n}$ (the encoder or sensor in our inverse problem). This is viewed as assigning $n$ parameters to $f$. The second mapping $M$ takes any point $y$ in $\mathbb{R}^{n}$ and assigns to it a function $M(y) \in L_{2}$ (the decoder). The set of all images $M(y), y \in \mathbb{R}^{n}$, is viewed as an $n$ dimensional manifold.

To approximate a given $f \in \mathcal{F}$, we use $M(a(f))$ which is a point on this manifold. The manifold width $\delta_{n}\left(\mathcal{F}, L_{2}\right)$ is then defined as the best performance one can obtain by such a scheme:

$$
\delta_{n}\left(\mathcal{F}, L_{2}\right):=\inf _{a, M} \sup _{f \in \mathcal{F}}\|f-M(a(f))\|_{L_{2}},
$$

where the infimum is taken over all such continuous mappings $a, M$ of the above form for the fixed value of $n$.

Upper and lower bounds for the manifold widths of Sobolev and Besov classes are given in $[4,5]$. For our compact set $\mathcal{F}_{r}$, it is known that (see [4])

$$
\delta_{n}\left(\mathcal{F}_{r}, L_{2}\right) \geq c_{r} n^{-r}
$$

with $c_{r}$ a constant depending at most on $r$.

In order to show that this lower bound applies in our problem, we need to check that any temperature measuring algorithm can be described by such mappings $a$ and $M$. For $a$ we take the mapping of $f$ into the values $\left(x_{0} ; t_{1}, \ldots, t_{n} ; u_{1}, \ldots, u_{n}\right)$. Thus $a$ maps $\mathcal{F}_{r}$ into $\mathbb{R}^{2 n+1}$. This covers both fixed times and adaptive times as long as the adaptive choice of times is continuous with respect to varying $f$.

To make sure that this mapping $a$ is continuous, we need to see that the $u_{j}$ depend continuously

on $f$. To check this, let $f$ and $\bar{f}$ be any two functions from $\mathcal{F}_{r}$ and $\hat{f}_{k}$ and $\hat{f}_{k}$ be their Fourier 
coefficients. Then,

$$
\|\bar{f}-f\|_{L_{2}}^{2}=\frac{\pi}{2} \sum_{k=1}^{\infty}\left|\hat{f_{k}}-\hat{f}_{k}\right|^{2}
$$

Hence,

$$
\left|\bar{u}_{j}-u_{j}\right| \leq \sum_{k=1}^{\infty}\left|\hat{f_{k}}-\hat{f}_{k}\right| e^{-k^{2} t_{j}} \leq \sqrt{2 / \pi}\|\bar{f}-f\|_{L_{2}}\left\|\left(e^{-k^{2} t_{1}}\right)_{k=1}^{\infty}\right\|_{\ell_{2}} \leq C_{1}\|\bar{f}-f\|_{L_{2}},
$$

where $C_{1}$ depends only on the smallest value $t_{1}$ of the time sequence. Since for any measurement algorithm, the first time $t_{1}$ is fixed, we see that the mapping $a$ is continuous.

We can now formulate the following result

Theorem 3.1 For any measurement algorithm (fixed or adaptive) with a continuous decoder $A_{n}$, we have that

$$
\hat{\delta}_{n}\left(\mathcal{F}_{r}, L_{2}\right) \geq \delta_{n}\left(\mathcal{F}_{r}, L_{2}\right) \geq c_{r} n^{-r}
$$

where $c_{r}$ is given in (3.2).

Proof: We have shown above that any such measurement algorithm falls into the class of admissible mappings in the definition of the manifold width $\delta_{n}\left(\mathcal{F}, L_{2}\right)$ and so the first inequality is by definition of the manifold width. The second inequality is (3.2).

\section{Time selection for near optimal recovery}

We now show that placing a thermometer at the position $x_{0}$ of $\S 2$ (an algebraic number of degree two) allows us to take measurements at later times $t_{1}<t_{2}<\cdots<t_{n}$ such that from these $n$ measurements, we can recover $f$ at the optimal rate $n^{-r}$. We will actually prove slightly more since we will create one sequence $t_{1}<t_{2} \cdots$, such that for each $n$, choosing the first $n$ of these times achieves the desired result.

The main result of this paper is as follows:

Theorem 4.1 Consider the solution $u(x, t)$ to the heat equation (1.1) with initial condition $f$. We fix $x_{0}$ fullfiling (2.3) and an initial time $t_{1}>0$ in an arbitrary way. We then sample $u$ at $x_{0}$ and at times $t_{j}:=\rho^{j} t_{1}$, where $\rho=2 \sqrt{2}$. Then, whenever $f \in \mathcal{F}_{r}, r>0$, we can use these first $n$ values to construct an approximation $F_{n}$ to $f$ that satisfies

$$
\left\|f-F_{n}\right\|_{L_{2}[0, \pi]} \leq C\left(r, t_{1}\right) n^{-r}, \quad n \geq 1 .
$$

Note that the algorithm we have chosen is non-adaptive. Before getting into the proof, let us briefly describe the general strategy, which leads naturally to the above choice of the sampling time-instances.

We will use the time samples $u_{j}$ to create an approximation $\bar{f}_{k}$ to the Fourier coefficient $\hat{f}_{k}$, $k=1, \ldots, n$. Given such approximate coefficients, the function

$$
\bar{f}(x):=\sum_{k=1}^{n} \bar{f}_{k} \sin k x
$$


gives an approximation error (see (3.3))

$$
\begin{aligned}
\frac{2}{\pi}\|f-\bar{f}\|_{L_{2}}^{2} & \leq \sum_{k=1}^{n}\left|\hat{f}_{k}-\bar{f}_{k}\right|^{2}+\sum_{k \geq n+1}\left|\hat{f}_{k}\right|^{2} \\
& \leq \sum_{k=1}^{n}\left|\hat{f}_{k}-\bar{f}_{k}\right|^{2}+n^{-2 r} \sum_{k \geq n+1} k^{2 r}\left|\hat{f}_{k}\right|^{2} \leq \sum_{k=1}^{n}\left|\hat{f}_{k}-\bar{f}_{k}\right|^{2}+n^{-2 r},
\end{aligned}
$$

where we have used the definition (1.5) in the last inequality. This means that we want each $\bar{f}_{k}$ to approximate $f_{k}$ to sufficient accuracy so that the sum remaining on the right side can be bounded by $\mathrm{Cn} n^{-2 r}$.

To uncover the properties we want of the time sequence, we introduce the function

$$
F(t):=\sum_{k=1}^{\infty} c_{k} e^{-k^{2} t}, \quad c_{k}:=\hat{f}_{k} \sin k x_{0}
$$

which is defined for $t \in(0, \infty)$. The value of $u\left(x_{0}, t_{j}\right)$ is the same as $F\left(t_{j}\right)$. We first consider the problem of approximating the coefficients $c_{k}$ from the values of $F\left(t_{j}\right), j=1, \ldots, n$. Later we relate this back to our problem of approximating $f$.

Let $t_{1}<t_{2}<\cdots t_{n}$ be any increasing sequence of $n$ times. We want to derive sufficient conditions on this sequence so that from the values $F\left(t_{j}\right), j=1, \ldots, n$, we can recover the coefficients $c_{k}, k=$ $1, \ldots, n$, to sufficiently high accuracy. We shall use the sample at $t_{n}$ to compute an approximation $\bar{c}_{1}$ to $c_{1}$ and then in general use $t_{n-k+1}$ to construct an approximation $\bar{c}_{k}$ to $c_{k}$. For each $k$, we can write

$$
c_{k}=e^{k^{2} t_{n-k+1}} F\left(t_{n-k+1}\right)-\sum_{j=1}^{k-1} c_{j} e^{\left(k^{2}-j^{2}\right) t_{n-k+1}}-\sum_{j>k} c_{j} e^{-\left(j^{2}-k^{2}\right) t_{n-k+1}} .
$$

We define $\bar{c}_{1}:=e^{t_{n}} F\left(t_{n}\right)$ and then recursively define

$$
\bar{c}_{k}:=e^{k^{2} t_{n-k+1}} F\left(t_{n-k+1}\right)-\sum_{j=1}^{k-1} \bar{c}_{j} e^{\left(k^{2}-j^{2}\right) t_{n-k+1}}, \quad k=2, \ldots, n .
$$

It follows that

$$
c_{k}-\bar{c}_{k}=\sum_{j=1}^{k-1}\left[\bar{c}_{j}-c_{j}\right] e^{\left(k^{2}-j^{2}\right) t_{n-k+1}}-\sum_{j>k} c_{j} e^{-\left(j^{2}-k^{2}\right) t_{n-k+1}}, \quad k=1,2, \ldots, n .
$$

Let us denote by $E_{j}:=\left|c_{j}-\bar{c}_{j}\right|$, the error by which we recover each $c_{j}, j<k$. We have

$$
E_{1} \leq \sum_{j \geq 2} j^{-r} e^{-\left(j^{2}-1\right) t_{n}} \leq 2^{-r} e^{-3 t_{n}} \sum_{j \geq 2} e^{\left(-j^{2}+4\right) t_{1}} \leq A_{0} e^{-3 t_{n}}
$$

with

$$
A_{0}:=A_{0}\left(t_{1}\right):=\sum_{j=0}^{\infty} e^{-j t_{1}}=\left(1-e^{-t_{1}}\right)^{-1}
$$


a constant depending only on the initial choice $t_{1}$. In general, formula (4.5) gives

$$
E_{k} \leq \sum_{j=1}^{k-1} e^{\left(k^{2}-j^{2}\right) t_{n-k+1}} E_{j}+\sum_{j=k+1}^{\infty} j^{-r} e^{-\left(j^{2}-k^{2}\right) t_{n-k+1}}=: \Sigma_{1}(k)+\Sigma_{2}(k), \quad k \geq 2 .
$$

Let us first bound the sum $\Sigma_{2}(k)$. We have that

$$
\begin{aligned}
\Sigma_{2}(k) & \leq(k+1)^{-r} \sum_{j=k+1}^{\infty} e^{-(j+k) t_{n-k+1}}=(k+1)^{-r} e^{-(2 k+1) t_{n-k+1}} \sum_{j=0}^{\infty} e^{-j t_{n-k+1}} \\
& \leq(k+1)^{-r} e^{-(2 k+1) t_{n-k+1}}\left(1-e^{-t_{1}}\right)^{-1}=A_{0}(k+1)^{-r} e^{-(2 k+1) t_{n-k+1}} .
\end{aligned}
$$

We will now give a choice of the $t_{j}$ so that we can derive a bound for $\Sigma_{1}(k)$ comparable to the right side of (4.11) and that when we combine this bound with the bound for $\Sigma_{2}(k)$ given in (4.11) we obtain a good bound for the error $E_{k}$. We want to keep the description of the $t_{j}$ simple although more exotic choices would give more favorable bounds on their growth.

The following lemma provides a choice of sampling time-instances ensuring optimal recovery.

Lemma 4.2 Given any fixed choice of $t_{1}$, we define $\rho:=2 \sqrt{2}$ and inductively define

$$
t_{k}:=\rho^{k-1} t_{1}, \quad k \geq 2 .
$$

For this choice of the sequence $\left(t_{k}\right)$ we have

$$
E_{k} \leq A_{0} 2^{k} e^{-(2 k+1) t_{n-k+1}}, \quad k=1,2, \ldots
$$

Proof: We will prove (4.13) by induction. From (4.8), we know that (4.13) is valid for $k=1$. Suppose that we have already shown that $E_{j} \leq A_{0} 2^{j} e^{-(2 j+1) t_{n-j+1}}$ for $j<k$. We know that $\Sigma_{2}(k) \leq(k+1)^{-r} A_{0} e^{-(2 k+1) t_{n-k+1}}$ and so we are left with estimating $\Sigma_{1}(k)$ for this choice of the sequence $\left(t_{j}\right)_{j \geq 1}$. In this direction, we have

$$
\Sigma_{1}(k) \leq \sum_{j=1}^{k-1} A_{0} 2^{j} e^{\left(k^{2}-j^{2}\right) t_{n-k+1}} e^{-(2 j+1) t_{n-j+1}} \leq A_{0} \sum_{j=1}^{k-1} 2^{j} e^{\left\{k^{2}-j^{2}-(2 j+1) \rho^{k-j}\right\} t_{n-k+1}} .
$$

Using (4.11), it follows that

$$
E_{k} \leq A_{0} e^{-(2 k+1) t_{n-k+1}}\left\{(k+1)^{-r}+\sum_{j=1}^{k-1} 2^{j} e^{\left\{k^{2}-j^{2}-(2 j+1) \rho^{k-j}+(2 k+1)\right\} t_{n-k+1}}\right\} .
$$

We will now show that the term in curly brackets is bounded by $2^{k}$.

We start with the following claim:

$$
k^{2}-j^{2}+(2 k+1)=(k+1-j)(k-j+2 j+1) \leq(2 j+1) \rho^{k-j}, \quad 1 \leq j \leq k-1 \text { and } k=2,3, \ldots
$$

To prove this, we note first that

$$
(k+1-j) \leq 2^{k-j}, \quad 1 \leq j \leq k-1 \text { and } k=2,3, \ldots,
$$


which follows from the fact that $m+1 \leq 2^{m}, m \geq 1$. Secondly, we note that

$$
\frac{k-j+2 j+1}{2 j+1} \leq 1+\frac{k-j}{3} \leq 2^{(k-j) / 2}, \quad 1 \leq j \leq k-1 \text { and } k=2,3, \ldots,
$$

which follows from the fact that $1+m / 3 \leq 2^{m / 2}, m \geq 1$. Combining (4.17) with (4.18), we obtain (4.16).

We now use (4.16) in (4.15) and obtain

$$
E_{k} \leq A_{0} e^{-(2 k+1) t_{n-k+1}}\left\{(k+1)^{-r}+\sum_{j=1}^{k-1} 2^{j}\right\} \leq A_{0} 2^{k} e^{-(2 k+1) t_{n-k+1}},
$$

as desired.

Now that the lemma is proved, the proof of the main theorem can be completed.

Proof of Theorem 4.1: From the sampled values, we can compute the approximation $\bar{c}_{k}$ to $c_{k}:=\hat{f}_{k} \sin k x_{0}$ given in (4.6). From Lemma 4.2, we know that

$$
\left|c_{k}-\bar{c}_{k}\right| \leq A_{0} 2^{k} e^{-(2 k+1) t_{n-k+1}}, \quad 1 \leq k \leq n .
$$

It follows from $(2.3)$ that for $\bar{f}_{k}:=\bar{c}_{k} / \sin k x_{0}$, we have

$$
\left|\hat{f}_{k}-\bar{f}_{k}\right| \leq C\left(t_{1}\right) k 2^{k} e^{-(2 k+1) t_{n-k+1}}, \quad 1 \leq k \leq n .
$$

We define $F_{n}:=\sum_{k=1}^{m} \bar{f}_{k} \sin k x$, with $m:=\lceil n / 2\rceil$. Then, from (4.3), with $C$ a constant depending only on $t_{1}$ and $r$ (where the value of $C$ may change at each appearance), we derive

$$
\begin{aligned}
\left\|f-F_{n}\right\|_{L_{2}[0, \pi]}^{2} & \leq C \sum_{k=1}^{m} k^{2} 2^{2 k} e^{-2(2 k+1) t_{n-k+1}}+m^{-2 r} \\
& \leq C e^{-6 t_{1} \rho^{n / 2}} \sum_{k=1}^{m} e^{2 \ln k+2 k \ln 2}+m^{-2 r} \\
& \leq C e^{-6 t_{1} \rho^{n / 2}} \sum_{k=1}^{n} e^{(2+2 \ln 2) k}+m^{-2 r} \\
& \leq C n e^{(2+2 \ln 2) n} e^{-6 t_{1} \rho^{n / 2}}+m^{-2 r} \\
& \leq C n^{-2 r} .
\end{aligned}
$$

Here, we have used the fact that $\ln k \leq k / 2$ for $k \geq 1$ in deriving the third inequality. For the last inequality, we observe that for any fixed $t_{1}$, we have $n e^{(2+2 \ln 2) n} e^{-6 t_{1} \rho^{n / 2}} \leq C n^{-r}$ for $n$ sufficiently large (depending only on $t_{1}$ and $r$ ).

Remark 4.3 The above theorem holds equally well if $\mathcal{F}^{r}$ is taken as the unit ball of a Besov space $B_{q}^{r}\left(L_{2}\right)$. In this case, it is known that (4.3) remains valid and the analysis of the first term is the same. 


\section{Final remarks}

We have introduced the problem of finding the initial temperature profile in heat propagation from later temperature measurements. We have only considered this problem in the simple univariate setting of heat conduction in a rod.

We have focused on the case of a single thermometer but that of multiple ones is also of interest and needs further investigation. Note however that if, at different time instances $t_{j}$, measurements are done at different locations, $x_{j}$ all sharing the same property of being algebraic of degree two and sharing (2.3) with the same lower bound, then our main result applies in that case too.

As mentioned above, it would be of interest to analyze the same question when the thermometer is placed in strategic points not fulfilling the lower bound (2.3). Obviously, it is also of interest also to study this problem for conduction on domains in higher dimensions. But, if stated exactly as in this paper, the problem is then underdetermined. It is then natural to take measurements not only at points $x_{0}$ but on manifolds of higher dimensions, or even of balls of small size describing the support of the thermometer.

In several space dimensions, the results will depend on the geometry of the body. Indeed, the eigenfunctions for the heat conduction problem will now depend on this geometry and this has a direct effect on the space position of the measurements. For example, the analogue of statement (2.3) comes into question. In the multi-dimensional cases there are mainly two types of results that one could expect. On one hand, to analyze specific geometries (the square, the disk,...) in which the spectrum is explicitly known. Note however, that even if the analogue of (2.3) were guaranteed in several space dimensions we have the added difficulty that, according to Weyl's Theorem, the spectrum of the Laplacian grows like $\lambda_{k} \sim c k^{2 / d}, d$ being the space dimension. Thus, in $d=2$ and higher, the series associated to the inverse of the eigenvalues diverges, contrarily to what occurs in one dimension where $\lambda_{k}=k^{2}$. It is well known that this fact has an important impact of the type of recovery results one might expect ([10]). The same could occur if the rod under consideration would have infinite length.

All our work is based on the Fourier series expansions. Very likely our analysis could be extended to more general linear one dimensional heat equations with space-dependent Fourier coefficients for which a Fourier representation formula applies and there is sufficient knowledge of the spectrum (see [12]). But our techniques cannot be employed for linear equations with time-dependent coefficients, nor in the nonlinear setting. It would be interesting to see if statements such as those in Theorem 4.1 can still be established. Note that the conclusion of Theorem 4.1 itself does not require a Fourier representation formula and that could be a natural goal in this more general setting. We emphasize however that our proof heavily relies on the possibility of using Fourier representation.

Even in the simple setting of a rod just considered, there are questions on the optimality of the selection of sample points. Observe that in our construction the sequence $\left(t_{j}\right)$ has been chosen once and for all independent of $n$ or $r$ and even of $f$. In this sense the choice is universal and the algorithm we develop is non-adaptive. It would be interesting to see what else could be done employing adaptive algorithms.

We conclude this section and the paper with some specific questions:

Q1 Growth of $t_{j}, j \rightarrow \infty$ : In Theorem 4.1 have shown that we can take $t_{j} \sim \rho^{j}$ with $\rho=2 \sqrt{2}$. One can easily see the value of $\rho$ can be improved slightly. It is of interest to know whether exponential growth of the $t_{j}$ is necessary. 
Q2 Time choices dependent on $n$ : We have given a universal sequence $\left(t_{j}\right)$ such that for each $n$ the choice $t_{1}, \ldots, t_{n}$ gives optimal rate performance. One can ask whether there is a benefit to allow the selection of the time points to depend more strongly on $n$, namely not to be drawn from a universal sequence.

Q3 Finite time observations: Suppose we are restricted to make our time observations over a finite interval $[0, T]$. What is the optimal performance we can expect? As we have noted earlier, all of our estimates depend on the allowable first measurement $t_{1}>0$. If $t_{1}$ is specified then one can determine the maximum $n$ such that $t_{1} \rho^{n} \leq T$ and therefore obtain the bound $C\left(t_{1}, r\right) n^{-r}$ for the error in recovering $f$. However, now Q2 becomes more relevant.

Q4 Lower bounds: It would be interesting to establish lower bounds for the growth of the sequence $\left(t_{j}\right)$ necessary for establishing the optimal asymptotic decay for the classes $\mathcal{F}_{r}$.

Q5 Noisy observations: Recovery schemes should be developed and analyzed for noisy observations of $u\left(x_{0}, t_{j}\right), j=1, \ldots, n$. This appears to require substantially more analysis even in the simple case of a rod that we have discussed. In our recover method, the multiplication by $e^{k^{2} t_{n-k+1}}$ at each iteration would give a tremendous amplification of the noise. This would lead one to anticipate that our algorithm should be completely redesigned in the noisy setting.

Acknowledgements. The authors acknowledge Mourad Choulli for fruitful discussions and valuable bibliographical comments.

\section{References}

[1] I.V. Back, B. Blackwell, and C.R. St. Claire, Inverse Heat Conduction, Wiley, 1985.

[2] J. R. Cannon, The one-dimensional heat equation, Encyclopedia of Mathematics and its Applications, 23. Addison-Wesley Publishing Company, Advanced Book Program, Reading, MA, 1984. $\mathrm{xxv}+483 \mathrm{pp}$.

[3] M. Choulli, Une introduction aux problèmes inverses elliptiques et paraboliques, Mathématiques \& Applications, 65, Springer, 2009.

[4] R. DeVore, R. Howard, and C. Micchelli, Optimal nonlinear approximation, Manuscripta Math., 63 (1989), 469-478.

[5] R. DeVore, G. Kyriazis, D. Leviatan, and V. Tikhomirov, Wavelet compression and nonlinear n-widths, Advances in Computational Math., 1 (1993) 197-214.

[6] M. Fabrizio, A. Morro, Mathematical Problems in Linear Viscoelasticity, SIAM Studies in Applied Mathematics, 12, 1992.

[7] F. John, Numerical solution of the equation of heat conduction for preceding times, Ann. Mat. Pura Appl., 40 (1955) 129-142.

[8] J. L. Lions, Pointwise control for distributed systems, in Control and Estimation in Distributed Parameter Systems, H. t. Banks, ed., Fronteirs in Applied Mathematics, 11, 1992, pp. 1-39. 
[9] J. M. Marbán, and C. Palencia, A new numerical method for backward parabolic problems in the maximum norm setting, SIAM J. Numer. Anal., 40 (4) (2002) 1405-1420.

[10] S. Micu and E. Zuazua. On the lack of null controllability of the heat equation on the half line. Transactions of the A.M.S., 353 (4) (2001), 1635-1659.

[11] J. W. Nunziato, On heat equations in materials with memory, Quartely Appl. Math., 29 (1971), pp. 187-204.

[12] J. Pöschel and E. Trubowitz, Inverse spectral theory, Pure and Applied Mathematics, 130. Academic Press, Inc., Boston, MA, 1987.

[13] J. D. Murray, Mathematical Biology, vol. 1 and vol. 2, Springer-Verlag, Berlim Heidelberg, 2002.

[14] S. Saitoh and M. Yamamoto, Stability of Lipschitz type in determination of initial heat distribution, J. Inequal. and Appl. 1 (1) (1997), 7383.

[15] D. V. Widder, An introduction to transform theory, Pure and Applied Mathematics, vol. 42, Academic Press Inc., 1971.

\author{
Ronald DeVore \\ Department of Mathematics \\ Texas A\&M University \\ College Station, TX 77840, USA \\ rdevore@math.tamu.edu \\ Enrique Zuazua \\ Basque Center for Applied Mathematics \\ 48009 Bilbao, Basque Country, Spain \\ and \\ Ikerbasque - Basque Foundation for Science \\ Plaza Bizkaia \\ 48011 Bilbao, Basque Country, Spain \\ zuazua@bcamath.org
}

